\title{
5th joint international conference on hyperfine interactions and symposium on nuclear quadrupole interactions (HFI/NQI 2014)
}

\author{
Wayne D. Hutchison • Andrew E. Stuchbery • \\ Heiko Timmers
}

Published online: 17 March 2015

(c) Springer International Publishing Switzerland 2015

These are the proceedings of the 5th Joint International Conference on Hyperfine Interactions and Symposium on Nuclear Quadrupole Interactions (HFI/NQI 2014). The conference, hosted by the University of New South Wales and the Australian National University, was held in Canberra, from 21-26 September 2014 at the spiritual home of Australian science, the uniquely designed Shine Dome of the Australian Academy of Sciences in the Australian capital. The meeting followed on from the four previous successful joint meetings in Bonn, Iguassu Falls, CERN and Beijing, and carried on an even longer conference tradition; HFI/NQI 2014 could also be termed the 17th International Conference on Hyperfine Interactions and the 21st International Symposium on Nuclear Quadrupole Interactions.

The conference organisation was facilitated by a local committee containing members from both universities together with the assistance of the Consec Conference Management team and with input from the associated international advisory and program committees as detailed below. The conference, formally opened by the Chief Executive Officer of the Australian Research Council, Professor Aidan Byrne, was attended by 95 delegates and accompanying persons. As with previous meetings, the attendees travelled from all parts of the world. Not only were delegates engaged by some excellent scientific presentations, as evidenced by the contents of these proceedings, but they also experienced the pleasant surrounds of spring time in Canberra. The conference excursion to Tibinbilla nature reserve for a close up encounter with some Australian wildlife, and then to the Canberra Deep Space Communication Complex, were a particular highlight.

Proceedings of the 5th Joint International Conference on Hyperfine Interactions and International Symposium on Nuclear Quadrupole Interactions (HFI/NQI 2014), Canberra, Australia, 21-26

September 2014

W. D. Hutchison $(\bowtie) \cdot$ H. Timmers

School of Physical, Environmental and Mathematical Sciences, The University of New South Wales,

Canberra, Australia

e-mail: W.Hutchison@adfa.edu.au

A. E. Stuchbery

Department of Nuclear Physics, Australian National University, Canberra, Australia 
The HFI/NQI conference series provides a forum for the discussion and publication of all aspects of hyperfine interactions, detected by nuclear radiations, and of all facets of nuclear quadrupole interactions, detected by resonance methods. The topical areas for the conference followed on from previous meetings with some modification. Invited speakers were largely nominated by the international advisory committee, with some input from the local organisers. Final selection of the invited presentations and of the contributed talks was done by the HFI/NQI 2014 program committee. In order to maximise opportunities for attendees to present their work, for the first time in the conference series, brief oral poster presentations were offered. Two poster sessions took place. Altogether there were 15 invited and 30 contributed talks, 9 brief oral poster presentations together with 56 poster presentations.

Finally we note that during the meeting the delegation paused to remember the passing of two important figures of the hyperfine interactions community in Klaus-Peter Lieb (Göttingen) and Dietmar Riegel (Berlin). Both colleagues were warmly commemorated and their contributions to the field and conference series were acknowledged. 


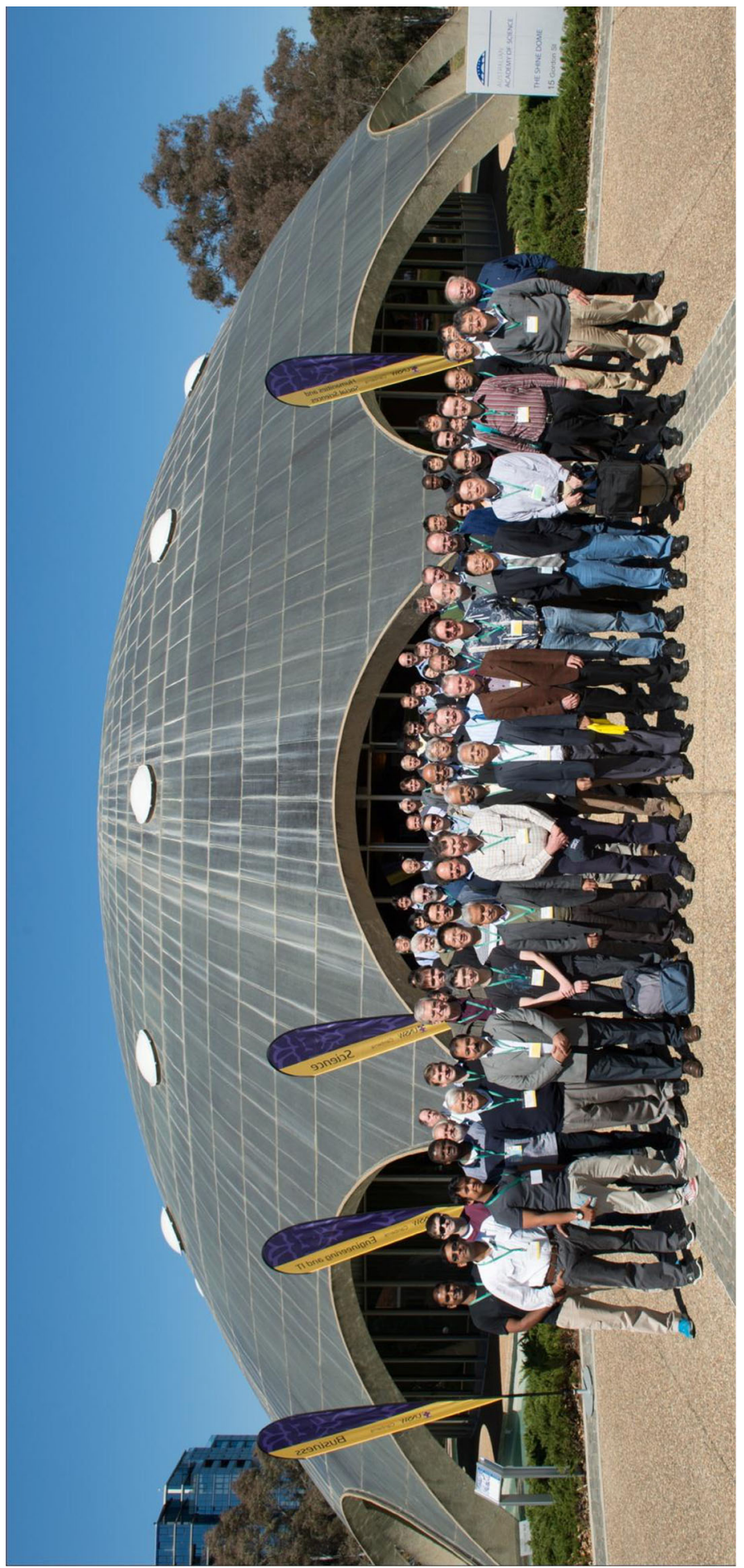

